\title{
Gene expression in Tribolium castaneum life stages: Identifying a species-specific target for pest control applications
}

\author{
Lindsey C Perkin ${ }^{\text {Corresp., } 1}$, Brenda Oppert ${ }^{2}$ \\ ${ }^{1}$ Southern Plains Agricultural Research Center, USDA, Agricultural Research Service, College Station, TX, United States \\ 2 Center for Grain and Animal Health Research, USDA, Agricultural Research Service, Manhattan, KS, United States \\ Corresponding Author: Lindsey C Perkin \\ Email address: lindsey.perkin@ars.usda.gov
}

The red flour beetle, Tribolium castaneum, is a major agricultural pest of post-harvest products and stored grain. Control of $T$. castaneum in stored products and grain is primarily by fumigants and sprays, but insecticide resistance is a major problem, and new control strategies are needed. T. castaneum is a genetic model for coleopterans, and the reference genome can be used for discovery of candidate gene targets for molecularbased control, such as RNA interference. Gene targets need to be pest specific, and ideally, they are expressed at low levels for successful control. Therefore, we sequenced the transcriptome of four major life stages of $T$. castaneum, sorted data into groups based on high or low expression levels, and compared relative gene expression among all life stages. We narrowed our candidate gene list to a cuticle protein gene (CPG) for further analysis. We found that the CPG sequence was unique to $T$. castaneum and expressed only in the larval stage. RNA interference targeting CPG in newly-emerged larvae caused a significant $(p<0.05)$ decrease in CPG expression $(1,491$-fold) compared to control larvae and $64 \%$ mortality over $18 \mathrm{~d}$. RNA-Seq of survivors after $18 \mathrm{~d}$ identified changes in the expression of other genes as well, including 52 long noncoding RNAs. Expression of three additional cuticle protein genes were increased and two chitinase genes were decreased in response to injection of CPG dsRNA. The data demonstrate that RNA-Seq can identify genes important for insect survival and thus may be used to develop novel biologicallybased insect control products. 
1 Gene expression in Tribolium castaneum life stages: Identifying a species-specific target for

\section{2 pest control applications}

3

4 Lindsey C. Perkin ${ }^{\S}$, and Brenda Oppert

5

6 USDA, Agricultural Research Service, Southern Plains Agricultural Research Center, 2881 F\&B

7 Road, College Station, TX 77845

8 USDA, Agricultural Research Service, Center for Grain and Animal Health Research, 1515

9 College Avenue, Manhattan, KS 66502

10

11

12 Running title: Tribolium castaneum life stage gene expression

13

14 §Corresponding author

15 Email addresses:

16 LCP: $\underline{\text { lindsey.perkin@ars.usda.gov }}$

17 BO: brenda.oppert@ars.usda.gov 


\section{Abstract}

20 The red flour beetle, Tribolium castaneum, is a major agricultural pest of post-harvest products

21 and stored grain. Control of T. castaneum in stored products and grain is primarily by fumigants

22 and sprays, but insecticide resistance is a major problem, and new control strategies are needed.

23 T. castaneum is a genetic model for coleopterans, and the reference genome can be used for

24 discovery of candidate gene targets for molecular-based control, such as RNA interference.

25 Gene targets need to be pest specific, and ideally, they are expressed at low levels for successful

26 control. Therefore, we sequenced the transcriptome of four major life stages of T. castaneum,

27 sorted data into groups based on high or low expression levels, and compared relative gene

28 expression among all life stages. We narrowed our candidate gene list to a cuticle protein gene

29 (CPG) for further analysis. We found that the CPG sequence was unique to T. castaneum and

30 expressed only in the larval stage. RNA interference targeting CPG in newly-emerged larvae

31 caused a significant $(\mathrm{p}<0.05)$ decrease in $\mathrm{CPG}$ expression $(1,491$-fold $)$ compared to control

32 larvae and $64 \%$ mortality over $18 \mathrm{~d}$. RNA-Seq of survivors after $18 \mathrm{~d}$ identified changes in the

33 expression of other genes as well, including 52 long noncoding RNAs. Injection of CPG dsRNA

34 also resulted in increased expression of three additional cuticle protein genes and reduced

35 expression of two chitinase genes in T. castaneum larvae. The data demonstrate that RNA-Seq

36 can identify genes important for insect survival and thus may be used to develop novel

37 biologically-based insect control products. 
40

41 The red flour beetle, Tribolium castaneum, is a pest of stored grain commodities. Traditional

\section{Introduction} control methods for $T$. castaneum and other stored product beetles are rapidly becoming less

effective, mostly because insect populations are developing resistance to pesticide treatments (Boyer et al. 2012). For example, storage pests around the globe are developing high resistance levels to one of the most common grain fumigants, phosphine (Opit et al., 2012; Pimentel et al., 2010). Thus, there is a need for new pest control strategies, and we are evaluating genetic-based treatments with target specificity and less damage to the environment, including the application of RNA interference (RNAi; Baum et al., 2007; Noh et al., 2012).

T. castaneum is a useful model to identify candidate genes because it has a sequenced genome (Tribolium Genome Sequencing Consortium, 2008) and responds well to RNAi when dsRNA is injected (RNAi; Brown et al., 1999; Tomoyasu \& Denell, 2004; Aronstein et al., 2011; Miller et al., 2012). Oral RNAi was successful in T. castaneum larvae against genes encoding vATPase (Whyard et al., 2009), inhibitor of apoptosis (Cao et al., 2018), and a voltage-gated sodium ion channel (Haim et al., 2016). However, we and others have not had success with oral RNAi in $T$. castaneum (unpublished data, Palli, 2014). Many factors may influence RNAi efficacy in insects, such as target sequence specificity, concentration and length of dsRNA, persistence of silencing effect in the target pest, and nucleases counteracting the effect of dsRNA (Huvenne \& Smagghe 2010; Allen \& Walker, 2012; Lomate \& Bonning, 2016; Guan et al., 2018, Cao et al., 2018). In the meantime, we have focused on the identification of gene targets with low expression requiring lower doses of dsRNA, and those that are expressed in critical feeding stages (larvae and adults) to improve the efficacy of oral RNAi. 
63

64 The iBeetle project (http://ibeetle-base.uni-goettingen.de) conducted a large-scale RNAi screen

65 in T. castaneum larvae and pupae with injected dsRNA, and various phenotypes were observed, 66 including mortality and developmental abnormalities (Schmitt-Engel et al., 2015). From this

67 screen, eleven genes were identified as potential pest control targets (Donitz et al., 2015; Ulrich 68 et al., 2015). These genes encode mostly products with GO terms related to the proteasome, and 69 mortality was observed after injection of larvae, pupae and adults. Some of these genes have 70 orthologs in other species, such as Drosophila melanogaster, Aedes aegypti, Apis mellifera, and 71 Acyrthosiphon pisum, and therefore RNAi or other molecular strategies based on these genes

72 may not be species-specific and may not be practical for insect control. Knorr et al. (2018)

73 selected 50 genes from the iBeetle study that resulted in $\geq 90 \%$ mortality to evaluate for RNAi in

74 Diabrotica vigifera vigifera and found that feeding adults dsRNA targeting 20 genes resulted in 75 mortality, and 36 retarded growth. While this work is important to highlight genes that have 76 application across species, the usefulness in the field may be limited due to off-target effects.

78 Previously, we applied RNA-Seq to the major developmental stages of T. castaneum to identify

79 highly expressed cysteine peptidase genes in feeding stages (larvae and adults) with likely

80 digestive functions (Perkin et al., 2016). A similar approach was used across the life stages of $D$.

81 melanogaster to reveal new transcribed regions and small non-coding RNAs, demonstrating that

82 transcriptome sequencing can discover new elements and help to annotate genomes of even the

83 most well studied insects (Graveley et al., 2011). Transcriptome sequencing of developmental

84 stages of the non-model oriental fruit fly, Bactocera dorsalis, and the harlequin bug, Murgantia 
85 histrionica, led to a list of candidate developmental and insecticide resistance genes for

86 downstream studies (Shen et al., 2011; Sparks et al., 2017).

87

88 In the present study, we examined gene expression patterns in T. castaneum life stages (adults,

89 eggs, larvae, and pupae), and used statistical analysis to sort expression patterns into groups of

90 low and high expression. Our hypothesis was that genes with low expression can be more

91 successfully targeted with RNAi or other applied control methods because the dose of dsRNA

92 required to knockdown transcript expression will be lower, and highly expressed genes and gene

93 expression patterns may be useful in understanding the physiology of the insect, or to identify

94 promoters for transgenic applications. Previously, we found that some genes with high

95 expression (i.e. those encoding cysteine peptidases) were important to insect physiology and

96 were duplicated in the genome, and complete knockdown of these genes induced a compensation

97 response and upregulation of other closely related genes (Perkin et al., 2016). Through screening

98 of potential targets in the present study, we identified a unique cuticle protein gene (CPG)

99 expressed in larvae, and we provide functional evidence for its potential as a molecular target for

100 pest control. We further evaluated the effect of CPG RNAi on the expression of other genes,

101 defining new potential metabolic connections between CPG and other genes.

102

103 Materials \& Methods

104 Insects

105 Insect strains and collection protocols were as previously described (Perkin et al., 2016).

106 Briefly, the T. castaneum lab colony used in this study was originally collected from a grain bin

107 in Kansas and has been maintained on $95 \%$ wheat flour and $5 \%$ brewer's yeast at $28^{\circ} \mathrm{C}, 75 \%$ 
108 R.H., 0L:24D. Insects were subcultured from the laboratory colony, and specific life stages were

109 removed. Adults were collected at 3-7 days post-eclosion and were mixed sex. Eggs were sifted

110 from diet 24-48 hours after oviposition and were separated from the fine particulate with a brush.

111 Larvae were collected at a late instar stage that was actively feeding (approximately 14 days post

112 hatch). Pupae included those with pigmentation in the eye, but not the elytra.

113

114 Library preparation and RNA sequencing of life stages

115 RNA was collected as three independent biological replicates from mixed sex groups of each life

116 stage (10 adults, approximately 500 eggs, 10 larvae, and 10 pupae per replicate). Tissue was

117 pulverized in TRIZOL (BulletBlender, Next Advance Inc., Averill Park, NY, USA) at speed 8

118 for 2 min with RNAse-free ziroconium oxide beads. RNA extraction and purification were with

119 a Zymo mini prep kit (Irvine, CA, USA). From the total RNA, DIRECTbeads (Agilient, Santa

120 Clara, CA, USA) were used to isolate polyA mRNA, and libraries were made with a $200 \mathrm{bp}$

121 RNA-Seq v2 kit (Life Technologies, Grand Island, NY, USA). Samples were sequenced on

122 318v2 chips on the Ion Torrent Personal Genome Machine (PGM, Life Technologies). Each run

123 provided approximately 1-5 million reads, with a total of 5-12 million reads per life stage (Perkin

124 et al., 2016). Life stage sequences were deposited at NCBI SRA as part of BioProject

125 PRJNA299695.

126

127 Data analysis

128 Relative gene expression was analyzed using ArrayStar (Lasergene Genomics Suite v14,

129 DNASTAR, Madison, WI, USA) by mapping reads to the NCBI Tcas5.2 genome build. Read

130 counts were normalized by Reads Per Kilobase of template per Million mapped reads (RPKM, 
131 Mortazavi et al., 2008). All data were filtered to groups with low expression (RPKM greater

132 than 2 less than $8 \log _{2}$; i.e., the $\log _{2}$ of the RPKM value was between 2 and 8 , not inclusive) and

133 high expression (RPKM greater than $8 \log _{2}$ ) for comparisons and biological screening. We use a

134 cutoff of RPKM greater than 2 to avoid underrepresented genes that can skew data.

136 Gene ontology (GO) terms were obtained and enrichment of GO terms was analyzed in

137 BLAST2GO PRO (version 4.1.5, Valencia, Spain, Götz et al., 2008). Over-representation tests

138 were performed with GO terms associated with genes in low and high categories in each life

139 stage, using the program g:Profiler and the g:GOSt function, which provides statistical

140 enrichment analysis (http://biit.cs.ut.ee/gprofiler/index.cgi; (Reimand et al., 2011). In g:Profiler,

141 results were limited to T. castaneum and the main GO categories: biological process (BP),

142 molecular function (MF) and cellular component (CC). Significance was determined using the

143 False Discovery Rate (FDR) multiple-testing correction threshold of 0.05 (Benjamini and

144 Hochberg 1995). Enriched GO terms also were obtained from the pairwise comparison of gene

145 expression in larvae injected with cuticle protein gene (CPG, LOC103313766) dsRNA compared

146 to mock-injected, where the test set was the significant $(\mathrm{p}<0.05)$ differentially expressed genes,

147 and the reference set was expression from all genes. GO terms were limited to $\mathrm{p}<0.05$ (after

148 FDR correction) and collapsed to the most specific description of each term.

\section{Candidate gene selection}

151 Genes were first filtered to only include those with expression levels between RPKM 2 and 8

$152\left(\log _{2}\right)$. The resulting gene list included 181 genes. We then filtered to only include genes

153 expressed at low levels in larvae because a field application would rely on genes expressed 
154 during the most active feeding stage, reducing the list to 139 genes. Our next filter was a

155 manually curated gene set and was based on GO analysis and gene annotations, selecting genes

156 with a function potentially necessary for survival.

157

158 The final filter submitted potential genes to the program OfftargetFinder (Good et al., 2016).

159 This program checks gene specificity by searching 101 arthropod transcriptomes, 21 mers at a

160 time, to the 1000 Insect Transcriptome Evolution (1KITE) dataset (http://www.1kite.org) and is

161 specifically designed to identify potential problems in designing dsRNA for insect control. The

162 algorithm provides visualization of specific regions of the target gene that may cause off-target

163 effects and allows for dsRNA to be designed to regions with increased specificity to the target

164 pest. This filter limited our gene list to only three genes. We selected CPG as our primary target

165 because the 3' region of CPG was unique to T. castaneum, and this region was used to design

166 primers for dsRNA.

167

$168 R N A i$

169 Primers for dsRNA targeting CPG were designed similar to Perkin et al. (2017a) via Primer-

170 BLAST (http://www.ncbi.nlm.nih.gov/tools/primer-blast/) using default parameters, and primer

171 specificity was evaluated against the T. castaneum genome. A primary set of primers was

172 designed to amplify CPG, and secondary primers were designed to amplify the 3 ' region to

173 minimize off-target effects..

174

175 The primary set of primers (Forward: ATAATCAAGCCCGTTTCCAACA, Reverse:

176 AATCACGACTACAAACATTCTTAGG) amplified CPG in $25 \mu 1$ PCR reactions using 
177 genomic DNA template from the lab strain $(2.5 \mu 1$ 10X buffer, $2.0 \mu \mathrm{l} 2.5 \mathrm{mM}$ dNTP, $0.5 \mu 110$

$178 \mu \mathrm{M}$ forward primer, $0.5 \mu \mathrm{l} 10 \mu \mathrm{M}$ reverse primer, $0.125 \mu 1$ JumpStart AccuTaq DNA

179 polymerase (Sigma-Aldrich, Saint Louis, MO, USA), $2 \mu 1$ genomic DNA, $17.375 \mu$ nuclease-

180 free water) and thermal cycle conditions as specified by the JumpStart AccuTaq DNA

181 polymerase product information guide (denaturation for $30 \mathrm{sec}$ at $95^{\circ} \mathrm{C}, 30$ cycles of $95^{\circ} \mathrm{C}$ for 30

$182 \mathrm{sec}$, primer annealing at $\mathrm{T}_{\mathrm{m}} 55.5^{\circ} \mathrm{C}$ for $30 \mathrm{sec}$, and extension at $68^{\circ} \mathrm{C}$ for $30 \mathrm{sec}$, final extension

183 at $68^{\circ} \mathrm{C}$ for $5 \mathrm{~min}$, and hold at $4^{\circ} \mathrm{C}$ ). The product was assessed on a $1 \%$ agarose E-gel

184 (ThermoFisher, Waltham, MA, USA) to ensure the amplified region was the correct size.

185

186 A second round of PCR was done with the secondary set of primers (Forward:

187 TATTCGTCTGTCGTCGCTCC, Reverse: AATCACGACTACAAACATTCTTAGG) for 188 specific targeting of CPG. The secondary PCR reaction was $100 \mu 1$ and the product from the

189 primary PCR reaction was used as the template $(10 \mu \mathrm{l} 10 \mathrm{X}$ buffer, $8 \mu \mathrm{l} 2.5 \mathrm{mM}$ dNTP, $2 \mu \mathrm{T} \mathrm{T} 7$

190 forward primer, $2 \mu \mathrm{l} \mathrm{T7}$ reverse primer, $0.5 \mu 1$ JumpStart AccuTaq DNA polymerase, $10 \mu 1$

191 primary PCR product, $67.5 \mu \mathrm{l}$ nuclease-free water). The thermal reaction profile was as follows:

192 denaturation for $30 \mathrm{sec}$ at $95^{\circ} \mathrm{C}, 5$ cycles of $95^{\circ} \mathrm{C}$ for $30 \mathrm{sec}$, primer $\mathrm{T}_{\mathrm{m}}$ for $30 \mathrm{sec}, 72^{\circ} \mathrm{C}$ for 30

$193 \mathrm{sec}, 29 \mathrm{cycles}$ of $95^{\circ} \mathrm{C}$ for $30 \mathrm{sec}$, primer $\mathrm{T}_{\mathrm{m}}$ plus $5^{\circ} \mathrm{C}$ for $30 \mathrm{sec}$, and $72^{\circ} \mathrm{C}$ for $30 \mathrm{sec}$, extension

194 for 5 min at $68^{\circ} \mathrm{C}$ and hold at $4^{\circ} \mathrm{C}$. The secondary PCR primers had a $\mathrm{T} 7$ construct attached to

195 the 5' end (TAATACGACTCACTATAGGG), and thus $5^{\circ} \mathrm{C}$ was added to the second round of

196 amplification as suggested in the kit manual. The secondary PCR products were checked again

197 via $1 \%$ agarose E-gel for appropriate length (150 bp) and sufficient amplification.

198 
199 PCR products from the secondary amplification were used to make dsRNA via MEGAscript T7

200 kit (ThermoFisher) according to the kit protocol ( $8 \mu \mathrm{l}$ dNTP mix, $2 \mu 1$ 10X reaction buffer, $8 \mu 1$

201 secondary PCR products with T7 construct, $2 \mu$ l enzyme mix), and after a $6 \mathrm{~h}$ incubation period

202 at $37^{\circ} \mathrm{C}$ with constant mixing, products were purified via MEGAclear kit (ThermoFisher). Size

203 and quantity were verified on a digital nanophotometer (Implen, Westlake Village, CA, USA)

204 and TapeStation (Agilent).

205

206

Micro-injected dsRA

207 Immediately before injection, dsRNA solution was mixed with blue dye (1:20) to aid in

208 visualization of the injected liquid as in Perkin et al. (2017a). Larvae used in injections were

209 briefly put on ice, transferred to a microscope slide with double-sided tape for stabilization, and

210 the entire slide was placed on top of a small ice block. Thirty individuals were injected with 200

211 ng of dsRNA in triplicate. CPG-dsRNA was injected into larvae weighing approximately $1 \mathrm{mg}$.

212 Each treatment injection had a corresponding control injection of dye only (Mock) and a non-

213 injection control (Control). Injections were with a Drummond Nanoject (Drummond Scientific

214 Co., Broomall, PA, USA) set at $69 \mathrm{nl}$ and fast injecting speed with a "bee-stinger" needle.

215 Needles were made with 3.5 Drummond glass capillary tubes (3-000-203-G) and a micropipette

216 puller (Sutter Instrument Co. Model P-97, Novato, CA, USA) using the program: heat 750, pull

217 100, velocity 8, time 250, and pressure 500. After injection, each group was allowed to recover

218 for $2 \mathrm{~h}$ at room temperature, and then were covered with diet (95\% wheat flour, 5\% brewer's

219 yeast) and kept at $28^{\circ} \mathrm{C}, 75 \%$ R.H., 0L:24D. All treatments and controls were followed for 18

220 days to monitor mortality and/or developmental abnormalities. 
222 Library preparation and RNA sequencing and data analysis after RNAi

223 We validated knockdown of CPG with RNA-Seq as previously (Perkin et al., 2017b). Eight

224 individuals from each treatment and control were flash frozen in liquid nitrogen on day 18 post

225 injection. Total RNA was extracted by Trizol (Thermo Fisher Scientific, Waltham, MA USA)

226 and Quick-RNA Mini Prep kit (Zymo Research, Irvine, CA USA). Libraries were made on the

227 NeoPrep (200 bp insert) and sequenced on a Mi-Seq (2x300, Illumina, San Diego, CA USA).

228 Three independent biological replicates of all groups and 3-4 technical replicates of CPG

229 dsRNA-injected larvae were sequenced (metrics are found in Supplemental File 5). Sequence

230 reads were deposited at NCBI SRA as PRJNA520884.

231 Arraystar (DNAStar, Madison, WI USA) was used to calculate RPKM and fold change between

232 treatment and control groups by mapping reads to the Tcas5.2 genome. We also used Arraystar

233 for statistical analysis, student t-tests between treatments and ANOVA among treatments. Genes

234 were filtered to only include those that had $>8$-fold difference between treatments, and

235 significant at $\mathrm{p}<0.01$ after FDR correction. To classify ncRNA, sequences were submitted to

236 RNAcon (website: http://crdd.osdd.net/raghava/rnacon/submit.html) for prediction using SVM

237 scores (default of 0.0; Panwar et al., 2014).

238

239 Cuticle protein tree

240 NCBI RefseqRNA was searched with term "cuticle protein" and limited to T. castaneum, which

241 returned a list of 160 genes which we then manually curated to only include relevant genes with

242 a chitin domain (pfam00379). This resulted in a total of 125 sequences in the final data set.

243 Protein sequences from the selected genes were used as input in MEGA X (version 10.0.5;

244 Kumar et al., 2018). Protein sequences were aligned using ClustalW. The evolutionary history 
245 of cuticle proteins was inferred using the Maximum Likelihood method, JTT matrix-based

246 model, and 500 bootstrap iterations (Jones et al., 1992), using the tree with the highest log

247 likelihood.

248

249 Results

250 In the following sections, transcriptome datasets of gene expression in four developmental stages

251 of $T$. castaneum were analyzed by sorting genes into categories of high and low expression

252 levels. Gene Ontology (GO) terms were associated with each group, and overrepresented terms

253 were identified by enrichment analysis. A cuticle protein gene was selected as a gene expressed

254 at low levels only in larvae and was evaluated via RNAi as a gene with critical function for

255 insect survival.

256

257 Life stage expression analysis - genes expressed at high levels

258 Analysis of $T$. castaneum genes with high expression levels (RPKM $\left.>8, \log _{2}\right)$ in all life stages

259 indicated there were 101, 290, 238, and 54 genes uniquely expressed in adults, eggs, larvae, and

260 pupae, respectively (Fig. 1, Supplemental File 1). Eggs and larvae stages shared the most

261 commonly expressed genes (162), followed by adults and larvae (48), and adults and eggs (29).

262 Genes common to eggs and larvae included 44 ribosomal proteins (large and small subunits),

263 indicative of the high degree of protein production occurring in these stages. Adults and larvae

264 feeding stages shared two digestive cathepsin L genes (LOC659441 and LOC659502; Martynov

265 et al., 2015; Perkin et al., 2016) and two chitinase precursor genes (Cht8 and Cht11). Only

266 LOC663906 encoding a single-stranded DNA-binding protein was commonly expressed in eggs

267 and pupae. Twelve genes were highly expressed in all stages, including those encoding two 
268 elongation factors, three ribosomal proteins, a cathepsin L (26-29-p), glyceraldehyde-3-

269 phosphase dehydrogenase 2, NADH dehydrogenase subunit 2, ATP synthase, cytochrome $\mathrm{c}$ and

270 cytochrome b, and I-rRNA (Table 1).

271

272 In the highly-expressed gene group, the top Molecular Function (MF) GO terms for each life

273 stage varied, but adults and larvae had shared term "nucleotide binding", whereas eggs and

274 larvae shared "hydrolase activity" (Fig. 2). "Oxidoreductase activity" was common to larvae,

275 pupae and adults. An enrichment analysis found $16 \mathrm{MF}$ terms belonging to "structural

276 constituent of the ribosome" $\left(\mathrm{p}=1.07 \times 10^{-11}\right)$ and 62 with "catalytic activity" $(\mathrm{p}=0.028)$ enriched

277 only in adults.

278

279 We also examined the most highly expressed genes in each developmental stage. The top 10

280 most highly expressed genes in adults included those encoding an odorant binding protein,

281 protamine, and cytochrome c oxidase subunit II (RPKM 7,694-20,300), and the highest

282 expressed genes in eggs had similar function, including chemosensory protein 2, I-rRNA and

283 cytochrome c oxidase subunit I and II (RPKM 7,960-26,611; Supplemental File 1). These genes

284 also were highly expressed in larvae, along with cathepsin L genes involved in digestion of

285 cereal proteins (RPKM 13,248-43,202; Martynov et al., 2015; Perkin et al., 2016). Highly

286 expressed genes in pupae encoded two cuticular proteins and a conserved cathepsin L, 26-29-p

287 (RPKM 3,576-13,818).

288

289 Life stage expression analysis - genes expressed at low levels 
290 A comparison of genes with low expression levels (RPKM between $2-8 \log _{2}$ ) revealed

291 expression of 273 genes unique to adults, 167 to eggs, 139 to larvae, and 14 to pupae (Fig. 3;

292 Supplemental File 2). Adults and larvae shared 138 genes including chitin deacetylase 7 (Cda7)

293 and two chitinase precursors (Cht4 and Cht9). Larvae and pupae shared a single gene,

294 LOC664054 encoding pathogenesis-related protein 5, which also was expressed in adults but at

295 higher levels and thus was not part of the low expression group. Nine genes expressed at low

296 levels were common across all life stages, four of which had uncharacterized functions (Table 1).

297 The other five included genes encoding maternal protein tudor (Tctud), poly(A) polymerase type

2983 , THO complex subunit 2, transmembrane protein 35 , and sentrin-specific protease.

299

300 A GO analysis of each group of unique genes indicated differences in top level functions among

301 stages, with more similar expression in the adults and larval stages, sharing MF terms "serine-

302 type endopeptidase activity", "oxidoreductase activity", and "transition metal ion binding" (Fig.

303 4). However, an enrichment analysis indicated that serine-type endopeptidases were

304 overrepresented only in the adults stage $(\mathrm{p}=0.005)$, and substrate-specific transporter activity was

305 overrepresented in the larval stage $(\mathrm{p}=0.04)$. Still, these two life stages shared 138 genes

306 expressed at low levels, not unexpected since both stages are active and feeding. Pupae had the

307 least number of genes and GO terms and included those with MF term "serine-type

308 endopeptidase activity", and two unique terms: "NADH dehydrogenase activity", and "structural

309 constituent of cuticle". Enrichment analysis for pupae indicated overrepresentation of negative

310 regulation of programmed cell death (BP; $\mathrm{p}=0.002)$, which was due to reads mapping to the gene

311 LOC663274 that encodes fas apoptotic inhibitory molecule 1, consistent with cell division and

312 tissue rearrangement occurring during this stage. Genes expressed in eggs were associated with 
313 terms "sequence-specific DNA binding" and "transcription factor activity sequence-specific

314 DNA binding". The most enriched categories in eggs were BP anatomical structure development

$315(p=0.002)$, sex determination $(p=0.011)$, and cell differentiation $(p=0.006)$, consistent with

316 highly complex developmental processes during embryogenesis.

318 Selection of candidate gene

319 To find candidate genes for RNAi, we manually curated genes unique to each life stage and 320 expressed at low levels (Supplemental File 2). Six cuticle genes were expressed only in larvae,

321 but unfortunately many cuticle genes have orthologs in other species. However, a BLAST of 322 CPG (LOC103313766) indicated no hits to other species (data not shown). In examining

323 previous data (Morris et al., 2009), CPG was found to be moderately expressed in the larval gut

324 (relative to other gut-expressed genes). Unfortunately, this gene was not analyzed by the iBeetle

325 project (Schmitt-Engel et al., 2015). We then submitted the sequence to OffTargetFinder and

326 found hits to only four other insect species which were focused to the 5 ' and middle regions,

327 leaving the 3' as a unique portion of the sequence (Supplemental Fig. 1). So, we chose

328 LOC103313766 CPG to evaluate as a potential candidate, since it met our criteria of uniquely

329 expressed in T. castaneum larvae, contained regions where dsRNA would minimize off target

330 effects, and was expressed in the larval gut.

331

332

RNAi validation of $C P G$

333 To demonstrate that this was a biological target, we used RNAi to knockdown expression of

334 CPG in T. castaneum larvae. We injected dsRNA into early stage larvae ( $2^{\text {nd }} 3^{\text {rd }}$ instar $)$, which

335 we hypothesized would have the most impact on transcript abundance. Knockdown and off

336 target effects were evaluated by another round of RNA-Seq on RNA isolated from the injected

Peer] reviewing PDF | (2018:11:32938:2:0:NEW 4 Apr 2019) 
337 larvae and controls (mock-injected "Mock" and noninjected "Control") $18 \mathrm{~d}$ post injection. The

338 RNAi treatment significantly reduced the expression of CPG target transcript 1,491-fold

339 compared to Control $\left(\mathrm{p}=2.17 \times 10^{-7}\right)$ and 284 -fold compared to Mock $\left(\mathrm{p}=7.14 \times 10^{-7}\right)$. An ANOVA

340 comparing the RPKM values of Mock, Control, and CPG treatments also was significantly

341 different (Fig. 5A; $\mathrm{p}=2.64 \times 10^{-5}$ ). The large decrease in expression was biologically significant

342 because $64 \%$ mortality was observed in the CPG dsRNA treatment group compared to the non-

343 injected control at day 18 post-injection (Fig. 5B; $\mathrm{p}=0.057$ ). We also observed 29\% mortality in

344 Mock, but it was not significant when compared to Control mortality $(19 \% ; p=0.79)$.

345

346 RNA-Seq analysis of larvae injected with CPG dsRNA compared to Mock resulted in 449

347 significantly differentially expressed genes $(\mathrm{p}<0.01$ and at least 8 -fold change; Supplemental Fig.

3482 and Supplemental File 3). Of those genes, 100 were uncharacterized, and 20 were annotated as

349 hypothetical proteins. Most (62\%) had decreased expression, and the 10 most highly repressed 350 genes were uncharacterized except the target gene and LOC100142553 adenylate kinase. Of

351 those with increased expression, the most highly increased was $J t b$, which encodes an orphan

352 receptor with unknown function, LOC662244 F-box SPRY that contributes to ubiquitin-protein

353 transferase activity, and BBIPl which is a chromosome associated protein. Other up regulated

354 genes related to transcription/translation included LOC107398339 (splicing factor for mRNA),

355 LOC103313738 (a repressor of developmentally regulated gene expression), and LOC664045,

356 involved in chromatin silencing. In response to knockdown of CPG, three other cuticle protein

357 genes were significantly increased: LOC100141875 cuticle 19-like, LOC103313752 cuticle

358 protein 70, and LOC655183 cuticle protein. 
360 There were 52 genes in this dataset that were annotated as noncoding RNA (ncRNA), 25 with

361 increased and 27 with decreased expression (Supplemental File 4). All were preliminarily

362 characterized as long ncRNAs since they were greater than $200 \mathrm{nt}$. All ncRNAs were screened

363 through RNAcon (Panwar et al., 2014) to predict the classification of each. There were 10 SSU-

364 rRNA5, three Intron-GP-1, and one IRESe; the remaining sequences were below the default

365 Support Vector Machines (SVM) threshold (0.0) and were classified as coding mRNA.

366 However, these sequences were characterized by NCBI as ncRNA, probably due to unusual

367 structure. Regardless, our transcriptome data suggests that ncRNA are actively transcribed in

368 response to RNAi, as has been described previously (Ji et al., 2015).

369

370 A GO term enrichment analysis of significantly decreased gene expression in response to CPG

371 RNAi identified functions involved in chitin breakdown (BP GO:0006032 and MF GO:0004568;

$372 \mathrm{p}=0.04$ and 0.04, respectively). LOC107398196 and Cht13, both encoding chitinases, were

373 responsible for these enriched GO terms. In contrast, the GO term aspartic-type endopeptidase

374 activity $\left(\mathrm{p}=8.28 \times 10^{-5}\right)$ was significantly enriched in up regulated genes.

375

376 Characterization of $C P G$

377 To gain insight into the function of the CPG target gene, a maximum likelihood tree was

378 constructed with T. castaneum protein sequences annotated as ‘cuticle protein' (Fig. 6). CPG

379 predicted protein (XP_008196095, highlighted with a yellow box) was most similar to cuticle

380 proteins 16.5 (XP_015833062) and 19.8 (XP_976285). Interestingly, the target CPG protein also

381 was found in the same major clade as the up regulated cuticle protein 70 gene LOC103313752

382 (XP_008196069, highlighted in red), and found in close proximity on LG3 (Table 2). Another 
383 cuticle protein gene with increased expression, LOC655183 (XP_008190752) also on LG3, was

384 related to those encoding resilin proteins. The protein product of the cuticle gene with highest

385 increased expression, LOC100141875 (XP_001809825), was related to cuticle protein 7

386 (XP_008193006) encoded by LOC656347, and both genes were found on LG5.

387

388 Discussion

389 Current control methods for T. castaneum and other stored product pests are becoming less

390 effective due to development of insecticide resistance (Collins, 1990; Jagadeesan et al., 2012;

391 Opit et al., 2012). Alternative management strategies are limited, and new molecular-based

392 controls are needed because they offer increased efficacy and selectivity, and targeted

393 approaches to combatting resistance. There are major hurdles in developing molecular-based

394 strategies in T. castaneum. The first step is to identify a target gene that can be effectively

395 knocked-down. The target gene should be specific to the pest or a narrow range of pests. The

396 most difficult challenge is delivery, whether it is topical or oral RNAi, or another mechanism. In

397 this study, we evaluated molecular targets by injection of dsRNA prior to attempts of topical or

398 oral delivery.

399

400 An RNA-Seq analysis of the four major life stages of $T$. castaneum identified twelve genes that

401 were highly expressed in all developmental stages. One gene was a cathepsin L named 26-29-p.

402 Cathepsin L genes in T. castaneum are part of a gene expansion group that are expressed at high

403 levels in adults and larvae for the digestion of cereal proteins (Goptar et al., 2012; Oppert et al.,

404 2010; Oppert et al., 2003; Oppert et al., 1993; Perkin et al., 2016; Perkin et al., 2017a;

405 Vinokurov et al., 2009). Based on phylogenetic analysis, 26-29-p is conserved across insects 
406 and may be the ancestral cathepsin L gene, but it most likely functions in development and

407 immunity (Perkin et al., 2016). While this gene may not be useful for species-specific pest

408 control because of the conservation of sequence among insects, its function in T. castaneum and

409 other insects is of biological interest, as it appears to provide critical function(s) throughout

410 development.

411

412 Nine genes were commonly expressed at low levels in all developmental stages, including Tctud

413 (maternal protein tudor). Tctud has been found in many eukaryotes and has been implicated in

414 protein-protein interactions, where methylated substrates bind to the tudor domain. In $D$.

415 melanogaster, tudor proteins are found in chromatin, small nuclear RNA assembly, RNA-

416 induced silencing complex, and germ granules (Ying \& Chen, 2012). Similar to Tctud, temporal

417 expression data shows low to moderately high expression of Dmtud in early fly embryos through

418 30-day old adults in both males and females (flybase.org, modENCODE temporal expression

419 data, Gramates et al., 2017). Additionally, mutants of various alleles caused lethality of early

420 embryos through maternal effects, and both male and female sterility (Thomson \& Lasko, 2004;

421 flybase.org, Summary of Phenotypes, Gramates et al., 2017). We submitted Tctud nucleotide

422 sequence to OffTargetFinder and found multiple 21 mer hits to 35 other species (data not shown), 423 and therefore the gene was not selected for RNAi.

425 We have found that validation of RNAi knockdown through RNA-Seq can provide unique 426 insights into function. Previously, we used RNA-Seq to validate knockdown of aspartate 1-

427 decarboxylase $(A D C)$ in $T$. castaneum larvae, resulting in the identification of additional gene

428 interconnectivity (Perkin et al., 2017b). Not only was the significant knockdown of $A D C$ 
429 confirmed through RNA-Seq, a previously unknown interaction between $A D C$ and dopamine

430 receptor 2 also was discovered. This information led to biological assays that determined that

431 the reduction of $A D C$ transcripts via RNAi resulted in adults with decreased mobility. Therefore,

432 we used the same approach in the present study to evaluate knock down of a target gene and also

433 understand the overall impact to the transcriptome of reduced gene expression.

434

435 To isolate a candidate gene that could be used as a molecular-based pest control target, we

436 sought genes unique to a feeding stage (i.e. larvae or adults) and expressed at low levels.

437 Through manual curation of life stage transcriptome data, we found CPG was uniquely expressed

438 in larvae at low levels and lacked an ortholog in other species at the 3' end of the gene; in fact,

439 BLAST did not return any hits at the mRNA level. We were encouraged that this target also has

440 potential as an oral RNAi product, since the gene was moderately expressed in the larval gut.

441 The protein product of this gene, XP_008196095, is a peptide of 61 amino acids, with $41 \%$

442 identity to a neuropeptide-like precursor from the flesh fly, Sacrophaga crassipalpis (Li et al.,

443 2009). Additionally, Bhatia and Bhattacharya (2018) found that knockdown of a related cuticle

444 protein gene in the green peach aphid (Myzus persicae) through oral delivery of dsRNA

445 expressed in Arabidopsis thaliana resulted in reduced fecundity. We did not investigate whether

446 the T. castaneum target cuticle protein also had neuropeptide properties or whether reduced gene

447 expression caused a reduction in fecundity, but our functional test with RNAi demonstrated that

448 decreased gene expression in early stage larvae resulted in significant mortality compared to

449 Control and Mock. Thus, this gene is a candidate target for oral delivery of dsRNA.

450 
451 RNA-Seq analysis of the transcriptome after RNAi knockdown of CPG identified three other

452 cuticle genes with increased expression and two chitinase genes with decreased expression

453 compared to Mock. The cuticle genes with increased expression included one encoding cuticle

454 protein 70 (XP_008196069), clustering in the same major clade as the target cuticle protein gene,

455 suggesting similar function and perhaps an indication of redundancy in function as has

456 previously been noted with gene expansion groups. In fact, this redundancy may explain

457 survivors in the dsRNA-injected larvae, albeit a low percentage (32\%), and it may complicate

458 oral RNAi if there is compensation response similar to that previously identified in attempting to

459 target cysteine protease genes via RNAi (Perkin et al., 2017a). Another cuticle protein gene

460 LOC655183 also was up regulated after RNAi treatment, and this gene product (XP_008190752)

461 clustered with resilin proteins. Resilin is a specialized cuticle protein that is found in soft parts

462 of the adult insect allowing for movement of wings (Andersen \& Weis-Fogh, 1964).

463 LOC655183 is an ortholog to a gene in Drosophila melanogaster encoding cuticular protein 56F,

464 which is expressed in the imaginal discs of late larvae (flybase.org, Gramates et al., 2017). The

465 protein product of another cuticle gene with increased expression, LOC100141875

466 (XP_001809825), clustered with cuticle protein 7 (XP_008193006), an ortholog to one found in

467 the carapace cuticle of juvenile horseshoe crab, Limulus Polyphemus with a high tyrosine content

468 (Ditzel et al., 2003).

469

470 Opposite in response to the increased expression of cuticle protein genes, Cht 13, a chitinase, was

471 decreased in response to the injection of CPG dsRNA. Cht13 is predicted to be part of group IV

472 chitinases expressed in the gut or fat body and expressed in response to feeding in larval and

473 adult stages (Zhu et al., 2008). All differentially expressed chitin-related genes are apparently 
474 interconnected through a regulatory pathway that responds to the loss of function of CPG by 475 RNAi.

476

477 We were surprised at the number of long ncRNAs (52) that were differentially expressed at a 478 significant level $(\mathrm{p}<0.01)$ in surviving T. castaneum larvae that were injected with CPG dsRNA.

479 Most ncRNA were not classified as typical ncRNAs and may represent new classes, or 480 alternatively may be miss-annotated. However, differentially expressed long ncRNA have been 481 implicated in human disease states, particularly cancer (Ma et al., 2015). Of relevance to this 482 study, differentially-expressed long ncRNAs can impose epigenetic changes that alter the 483 transcription of other genes, including silencing (Tufarelli et al., 2003). Therefore, we can 484 speculate that the large number of differentially expressed genes in this study (449) may have 485 been regulated in part by ncRNAs. While not to the same degree, ncRNAs were implicated in a 486 previous RNAi study, in which LOC107398253 ncRNA (decreased 12.3-fold, $\mathrm{p}=0.001$, in the 487 current study) was decreased 593-fold in response to RNAi of $A D C$ (Perkin et al., 2017b). This 488 particular ncRNA was annotated as an U3 snoRNA, predicted to be involved in site-specific 489 cleavage of ribosomal RNA (rRNA) during pre-rRNA processing (Clery et al., 2007). More 490 work is needed to understand the relationship of ncRNAs and RNAi in insects.

\section{Conclusions}

493 In summary, these data add to the sparse stage-specific studies in T. castaneum and other beetles.

494 We demonstrate that by using this approach, we were able to identify a candidate gene, CPG, 495 that may be useful in developing an insect control product based on dsRNA. Because the gene 
496 was expressed at low levels overall and moderate levels in the larval gut, oral delivery methods

497 for CPG dsRNA have the potential to control damage by T. castaneum larvae.

498

499 Acknowledgements

500 The authors would like to thank technicians Ken Friesen and Tom Morgan for their contribution,

501 injecting larvae and RNA extraction and library preparation, respectively. Mention of trade

502 names or commercial products in this publication is solely for the purpose of providing specific

503 information and does not imply recommendation or endorsement by the U.S. Department of

504 Agriculture. USDA is an equal opportunity provider and employer. 


\section{References}

506 Allen, M.L. and Walker, W.B., 3rd. 2012. Saliva of Lygus lineolaris digests double stranded ribonucleic acids. Journal of Insect Physiology 58: 391-396.

508

Andersen, S. O. and Weis-Fogh, T. 1964. Reslin. A rubberlike protein in arthropod cuticle. Advances in Insect Physiology 2:1-65.

Aronstein, K., Oppert, B. and Lorenzen, M. 2011. RNAi in agriculturally-important arthropods. In: RNA Processing (Grabowski, PP, ed.), pp. 157-180. InTech.

Baum, J.A., Bogaert, T., Clinton, W., Heck, G.R., Feldmann, P., Ilagan, O., Johnson, S., Plaetinck, G., Munyikwa, T., Pleau, M., Vaughn, T. and Roberts, J. 2007. Control of coleopteran insect pests through RNA interference. Nature Biotechnology 25: 1322-1326.

Benjamini, Y. and Hochberg, Y. 1995. Controlling the false discovery rate: a practical and powerful approach to multiple testing. Journal of Royal Statistics Society B Methodololgy 57:289-300.

Bhatia, V. and Bhattacharya, R. 2018. Host mediated RNAi of cuticular protein gene impaired fecundity in green peach aphid Myzus persicae. Pest Management Science. $10.1002 /$ ps.4900.

Boyer, S., Zhang, H. and Lemperiere, G. 2012. A review of control methods and resistance mechanisms in stored-product insects. Bullin Entomology Resources 102:213-29.

Brown, S.J., Mahaffey, J.P., Lorenzen, M.D., Denell, R.E., and Mahaffey, J.W. 1999. Using RNAi to investigate orthologous homeotic gene function during development of distantly related insects. Evolution and Development 1:11-15.

Cao, M., Gatehouse, J.A., and Fitches, E.C. 2018. A systematic study of RNAi effects and dsRNA stability in Tribolium castaneum and Acyrthosiphon pisum, following injection 
and ingenstion of analogous dsRNAs. International Journal of Molecular Sciences. 19: 1079, doi:10.3390/ijms19041079.

530 Clery, A., Senty-Segault, V., Leclerc, F., Raue, H.A. and Branlant, C. 2007. Analysis of

531

532

533

534 sequence and structural features that identify the B/C motif of U3 small nucleolar RNA as the recognition site for the Snu13p-Rrp9p protein pair. Molecular and Cell Biology 27:1191-1206.

Collins, P.J. 1990. A new resistance to pyrethroids in Tribolium castaneum (herbst). Journal of Pesticide Science 28:101-115.

Ditzel, N., Andersen, S. O. and Højrup, P. 2003. Cuticular proteins from the horseshoe crab, Limulus Polyphemus. Comparative Biochemistry and Physiology Part B 134(3):489-497.

Donitz, J., Schmitt-Engel, C., Grossmann, D., Gerischer, L., Tech, M., Schoppmeier, M., Klingler, M. and Bucher, G. 2015. iBeetle-Base: a database for RNAi phenotypes in the red flour beetle Tribolium castaneum. Nucleic Acids Research 43: D720-725.

El Halim, H.M.A., Alshukri, B.M.H., Ahmad, M.S., Nakasu, E.Y.T., Awwad, M.H., Salama, E.M., Gatehouse, A.M.R., and Edwards, M.G. 2016. RNAi-mediated knockdown of the voltage gated sodium ion channel $\mathrm{TcNa}_{\mathrm{v}}$ causes mortality in Tribolium castaneum. Scietific Reports 6:29301, doi:10.1038/srep29301.

Good, R.T., Varghese, T., Golz, J.F., Russell, D.A., Papanicolaou, A., Edwards, O. and Robin, C. 2016. OfftargetFinder: a web tool for species-specific RNAi design. Bioinformatics 32:1232-1234.

Goptar, I.A., Semashko, T.A., Danilenko, S.A., Lysogorskaya, E.N., Oksenoit, E.S., Zhuzhikov, D.P., Belozersky, M.A., Dunaevsky, Y.E., Oppert, B., Filippova, I.Y. and Elpidina, E.N. 2012. Cysteine digestive peptidases function as post-glutamine cleaving enzymes in 
551

552

553

554

555

556

557

558

559

560

561

562

563

564

565

566

567

568

569

570

571

572

tenebrionid stored-product pests. Comp Biochem Physiol B Biochem Mol Biol 161: 148154.

Götz, S., Garcia-Gomez, J.M., Terol, J., Williams, T.D., Nagaraj, S.H., Nueda, M.J., Robles, M., Talon, M., Dopazo, J. and Conesa, A. 2008. High-throughput functional annotation and data mining with the Blast2GO suite. Nucleic Acids Research 36:3420-3435.

Gramates, L.S., Marygold, S.J., Santos, G.D., Urbano, J.M., Antonazzo, G., Matthews, B.B., Rey, A.J., Tabone, C.J., Crosby, M.A., Emmert, D.B., Falls, K., Goodman, J.L., Hu, Y., Ponting, L., Schroeder, A.J., Strelets, V.B., Thurmond, J., Zhou, P. and the FlyBase, C. 2017. FlyBase at 25: looking to the future. Nucleic Acids Research 45:D663-D671.

Graveley, B.R., Brooks, A.N., Carlson, J.W., Duff, M.O., Landolin, J.M., Yang, L., Artieri, C.G., van Baren, M.J., Boley, N., Booth, B.W., Brown, J.B., Cherbas, L., Davis, C.A., Dobin, A., Li, R., Malone, J.H., Mattiuzzo, N.R., Miller, D., Sturgill, D., Tuch, B.B., Zaleski, C., Zhang, D., Blanchette, M., Dudoit, S., Eads, B., Green, R.E., Hammonds, A., Jiang, L., Kapranov, P., Langton, L., Perrimon, N., Sandler, J.E., Wan, K.H., Willingham, A., Zhang, Y., Zou, Y., Andrews, J., Bickel, P.J., Brenner, S.E., Brent, M.R., Cherbas, P., Gingeras, T.R., Hoskins, R.A., Kaufman, T.C., Oliver, B. and Celniker, S.E. 2011. The development transcriptome of Drosophila melanogaster. Nature 471:473-479.

Guan, R.B., Li, H.C., Fan, Y.J., Hu, S.R., Christiaens, O., Smagghe, G., and Miao, X.X 2018. A nuclease specific to lepidopteran insects suppresses RNAi. Journal of Biological Chemistry jbc.RA117.001553.

Huvenne, H. and Smagghe, G. 2010. Mechanisms of dsRNA uptake in insects and potential of RNAi for pest control: a review. Journal of Insect Physiology 56:227-235. 
573 Jagadeesan, R., Collins, P.J., Daglish, G.J., Ebert, P.R., Schlipalius, D.I. 2012. Phosphine

574 resistance in the rust red flour beetle, Tribolium castaneum (Coleoptera: Tenebrionidae):

575 inheritance, gene interactions and fitness costs. PloS one 7:e31582.

576 Ji, Z., Song, R., Regev, A. and Struhl, K. 2015. Many lncRNAs, 5'UTRs, and pseudogenes are

$577 \quad$ translated and some are likely to express functional proteins. eLife 4:e08890.

578 Jones D.T., Taylor W.R., and Thornton J.M. 1992. The rapid generation of mutation data

579 matrices from protein sequences. Computer Applications in the Biosciences 8: 275-282.

580 Knorr, E., Fishilevich, E., Tenbusch, L., Frey, M.L.F., Rangasamy, M., Billion, A., Worden,

581 S.E., Gandra, P., Arora, K., Lo, W., Schulenberg, G., Valverde-Garcia, P., Vilcinskas, A.

582 and Narva, K.E. 2018. Gene silencing in Tribolium castaneum as a tool for the targeted

583 identification of candidate RNAi targets in crop pests. Scientific Reports 8:2061,

584 doi:10.1038/s41589-018-20416-y.

585 Kumar S., Stecher G., Li M., Knyaz C., and Tamura K. 2018. MEGA X: Molecular Evolutionary 586 Genetics Analysis across computing platforms. Molecular Biology and Evolution $35: 1547-1549$.

Li, A., Rinehart, J.P. and Denlinger, D.L. 2009. Neuropeptide-like precursor 4 is uniquely expressed during pupal diapause in the flesh fly. Peptides 30:518-521.

Lomate, P.R. and Bonning, B.C. 2016. Distinct properties of proteases and nucleases in the gut, salivary gland and saliva of southern green stink bug, Nezara viridula. Scientific Report 6:27587.

Loktev, A.V., Zhang, Q., Beck, J.S., Searby, C.C., Scheetz, T.E., Bazan, J.F., Slusarski, D.C., Sheffield, V.C., Jackson, P.K. and Nachury, M.V. 2008. A BBSome subunit links ciliogenesis, microtubule stability, and acetylation. Developmental Cell 15:854-865. 
596 Ma, L., Li, A., Zou, D., Xu, X., Xia, L., Yu, J., Bajic V.B. and Zhang, Z. 2015. LncRNAWiki:

597 harnessing community knowledge in collaborative curation of human long non-coding $598 \quad$ RNAs Nucleic Acids Research 43:187-192.

599 Martynov, A.G., Elpidina, E.N., Perkin, L. and Oppert, B. 2015. Functional analysis of C1

600 family cysteine peptidases in the larval gut of Tenebrio molitor and Tribolium castaneum.

$601 \quad$ BMC Genomics 16:75.

602 Morris, K., Lorenzen, M.D., Hiromasa, Y., Tomich, J.M., Oppert, C., Elpidina, E.N., Vinokurov, 603 K., Jurat-Fuentes, J.L., Fabrick, J. and Oppert, B. 2009. Tribolium castaneum Larval Gut 604 Transcriptome and Proteome: A Resource for the Study of the Coleopteran Gut. Journal $605 \quad$ Proteome Research 8:3889-3898.

606 Miller, S.C., Miyata, K., Brown, S.J. and Tomoyasu, Y. 2012. Dissecting systemic RNA 607 interference in the red flour beetle Tribolium castaneum: parameters affecting the $608 \quad$ efficiency of RNAi. PLoS One 7:e47431.

609 Mortazavi, A., Williams, B.A., McCue, K., Schaeffer, L. and Wold, B. 2008. Mapping and 610 quantifying mammalian transcriptomes by RNA-Seq. Nature Methods 5:621-628.

611 Noh, M.Y., Beeman, R.W. and Arakane, Y. 2012. RNAi-based functional genomics in Tribolium 612 castaneum and possible application for controlling insect pests. Entomology Research $613 \quad 42: 1-10$

614 Noh, M.Y., Kramer, K.J., Muthukrishnan, S., Beeman, R.W., Kanost, M.R. and Arakane, Y. 615 2015. Loss of function of the yellow-e gene causes dehydration-induced mortality of 616 adult Tribolium castaneum. Developmental Biology 399:315-324. 
617 Opit, G.P., Phillips, T.W., Aikins, M.J. and Hasan, M.M. 2012. Phosphine resistance in

618 Tribolium castaneum and Rhyzopertha dominica from stored wheat in Oklahoma. Journal

619 of Economic Entomology 105:1107-1114.

620 Oppert, B., Elpidina, E.N., Toutges, M. and Mazumdar-Leighton, S. 2010. Microarray analysis

621 reveals strategies of Tribolium castaneum larvae to compensate for cysteine and serine

622 protease inhibitors. Comparitive Biochemistry and Physiology Part D Genomics

623 Proteomics 5:280-287.

624 Oppert, B., Morgan, T., Hartzer, K., Lenarcic, B., Galesa, K., Brzin, J., Turk, V., Yoza, K., Ohtsubo, K. and Kramer, K. 2003. Effects of proteinase inhibitors on digestive proteinases and growth of the red flour beetle, Tribolium castaneum (Herbst)(Coleoptera: Tenebrionidae). Comparitive Biochemistry and Physiology Part C Toxicology and Pharmacology 134:481-490.

Oppert, B., Morgan, T.D., Culbertson, C. and Kramer, K.J. 1993. Dietary mixtures of cysteine 630 and serine proteinase inhibitors exhibit synergistic toxicity toward the red flour beetle, Tribolium castaneum. Comparitive Biochemistry and Physiology Part C: Comparative Pharmacology 105:379-385.

Palli, S. R. 2014. RNA interference in Colorado potato beetle: steps toward development of dsRNA as a commercial insecticide. Current Opinion Insect Science 6:1-8.

Panwar, B., Arora, A. and Raghava, G.P.S. 2014. Prediction and classification of ncRNAs using structural information. BMC Genomics 15:127.

637 Perkin, L., Elpidina, E.N. and Oppert, B. 2016. Expression patterns of cysteine peptidase genes across the Tribolium castaneum life cycle provide clues to biological function. PeerJ

639 4:e1581. 
640 Perkin, L.C., Elpidina, E.N. and Oppert, B. 2017a. RNA interference and dietary inhibitors

641

642

643

644

645

646

647

648

649

650

651

652

653

654

655

656

657

658

659

660

induce a similar compensation response in Tribolium castaneum larvae. Insect Molecular Biology 26:35-45.

Perkin, L.C., Gerken, A.R., Oppert, B. 2017b. RNA-Seq validation of RNAi identifies additional gene connectivity in Tribolium castaneum (Coleoptera: Tenebrionidae). Journal of Insect Science 17:1-7.

Pimentel, M.A., D'A Faroni, L.R., Da Silva, F.H., Batista, M.D. and Guedes, R.N.C. 2010. Spread of phosphine resistance among Brazilian populations of three species of stored product insects. Neotropical Entomology 39:101-107.

Reimand, J., Arak, T. and Vilo, J. 2011. g:Profiler--a web server for functional interpretation of gene lists (2011 update). Nucleic Acids Research 39:W307-315.

Schmitt-Engel, C., Schultheis, D., Schwirz, J., Strohlein, N., Troelenberg, N., Majumdar, U., Dao, V.A., Grossmann, D., Richter, T., Tech, M., Donitz, J., Gerischer, L., Theis, M., Schild, I., Trauner, J., Koniszewski, N.D., Kuster, E., Kittelmann, S., Hu, Y., Lehmann, S., Siemanowski, J., Ulrich, J., Panfilio, K.A., Schroder, R., Morgenstern, B., Stanke, M., Buchhholz, F., Frasch, M., Roth, S., Wimmer, E.A., Schoppmeier, M., Klingler, M. and Bucher, G. 2015. The iBeetle large-scale RNAi screen reveals gene functions for insect development and physiology. Nature Communications 6:7822.

Sparks, M.E., Rhoades, J.H., Nelson, D.R., Kuhar, D., Lancaster, J., Lehner, B., Tholl, D., Weber, D.C. and Gundersen-Rindal, D.E. 2017. A Transcriptome Survey Spanning Life Stages and Sexes of the Harlequin Bug, Murgantia histrionica. Insects 8. 
661 Shen, G., Dou, W., Niu, J., Jiang, H., Yang, W., Jia, F., Hu, F., Cong, L. and Wang, J. 2011.

662 Transcriptome analysis of the Oriental fruit fly (Bactrocera dorsalis). PLoS one

$663 \quad 6: \mathrm{e} 29127$.

664 Thomson, T. and Lasko, P. 2004. Drosophila tudor is essential for polar granule assembly and 665 pole cell specification, but not for posterior patterning. Genesis 40:164-170.

666 Tomoyasu, Y. and Denell, R.E. 2004. Larval RNAi in Tribolium (Coleoptera) for analyzing 667 adult development. Developmental Genes and Evolution 214;575-578.

668 Tribolium Genome Sequencing Consortium. 2008. The genome of the model beetle and pest

669 Tribolium castaneum. Nature 452:949-955.

670 Tufarelli, C., Stanley, J.A., Garrick D, Sharpe, J.A., Ayyub, H., Wood, W.G. and Higgs, D.R. 671 2003. Transcription of antisense RNA leading to gene silencing and methylation as a 672 novel cause of human genetic disease. Nature Genetics 34:157-165.

673 Ulrich, J., Dao, V.A., Majumdar, U., Schmitt-Engel, C., Schwirz, J., Schultheis, D., Strohlein, N., Troelenberg, N., Grossmann, D., Richter, T., Donitz, J., Gerischer, L., Leboulle, G., Vilcinskas, A., Stanke, M. and Bucher, G. 2015. Large scale RNAi screen in Tribolium

Vinokurov, K.S., Elpidina, E.N., Zhuzhikov, D.P., Oppert, B., Kodrik, D. and Sehnal, F. 2009. Digestive proteolysis organization in two closely related Tenebrionid beetles: red flour reveals novel target genes for pest control and the proteasome as prime target. BMC beetle (Tribolium castaneum) and confused flour beetle (Tribolium confusum). Archives in Insect Biochemistry 70:254-279.

Whyard, S., Singh, A.D., and Wong, S. 2009. Ingested double-stranded RNAs can act as speciesspecific insecticides. Insect Biochemistsry and Molecular Biology 39:824-832. 
684 Ying, M. and Chen, D. (2012) Tudor domain-containing proteins of Drosophila melanogaster.

685 Developmental Growth \& Differentiation 54:32-43.

686 Zhu, Q., Arakane, Y., Banerjee, D., Beeman, R.W., Kramer, K.J., and Muthukrishnan, S. 2008.

687 Domain organization and phylogenetic analysis of the chitinase-like family of proteins in

688 three species of insects. Insect Biochemistry and Molecular Biology 38: 452-466 


\section{Table $\mathbf{1}$ (on next page)}

Genes that were expressed at high and low levels in all life stages.

List of genes that were expressed at high (top) and low (bottom) levels in all life stages of $T$. castaneum. 


\section{Tables}

2 Table 1. Genes that were expressed at high (top) and low (bottom) levels in all life stages of T. castaneum.

\begin{tabular}{|c|c|c|c|c|c|}
\hline \multirow[b]{2}{*}{ Name } & \multirow[b]{2}{*}{ Description } & \multicolumn{4}{|c|}{ Average RPKM } \\
\hline & & Adult & Egg & Larvae & Pupae \\
\hline LOC656235 & Elongation factor 1-gamma & 1,560 & 1,334 & 657 & 6,278 \\
\hline $\mathrm{RpS3}$ & Ribosomal protein $\mathrm{S} 3$ & 3,953 & 2,663 & 1,475 & 263 \\
\hline $26-29-p$ & Cathepsin L & 369 & 281 & 261 & 5,710 \\
\hline LOC659992 & 40S ribosomal protein $\mathrm{S} 29$ & 1,135 & 1,443 & 626 & 3,238 \\
\hline LOC663023 & Glyceraldehyde-3-phosphate dehydrogenase 2 & 2,405 & 812 & 2,135 & 2,375 \\
\hline LOC660435 & 60 S ribosomal protein L11 & 2,673 & 2,134 & 1,096 & 6,999 \\
\hline Efa1 & Elongation factor 1-alpha & 2,253 & 2,541 & 1,762 & 3,576 \\
\hline ND2 & NADH dehydrogenase subunit 2 & 1,081 & 1,457 & 1,000 & 401 \\
\hline ATP6 & ATP synthase F0 subunit 6 & 5,442 & 4,960 & 4,555 & 270 \\
\hline $\mathrm{COX} 3$ & Cytochrome c oxidase subunit III & 4,267 & 3,923 & 6,559 & 857 \\
\hline CYTB & Cytochrome b & 3,181 & 4,829 & 7,123 & 558 \\
\hline I-rRNA & I-rRNA & 16,635 & 11,824 & 34,216 & 11,020 \\
\hline LOC103315067 & Uncharacterized & 5.91 & 57.1 & 7.30 & 18.7 \\
\hline LOC 103312214 & Uncharacterized & 10.3 & 89.0 & 8.11 & 24.6 \\
\hline LOC 103315070 & Uncharacterized & 17.5 & 94.1 & 16.9 & 26.9 \\
\hline LOC107399196 & Uncharacterized & 25.5 & 181 & 9.69 & 22.7 \\
\hline LOC103312419 & Maternal protein tudor & 5.25 & 15.3 & 5.34 & 5.45 \\
\hline LOC 103312455 & poly(A) polymerase type 3 & 9.11 & 65.5 & 13.0 & 15.0 \\
\hline LOC663288 & THO complex subunit 2 & 7.55 & 61.8 & 8.69 & 10.6 \\
\hline LOC659780 & Transmembrane protein 35 & 240 & 7.72 & 241 & 179 \\
\hline LOC 103313244 & Sentrin-specific protease & 10.5 & 74.6 & 35.0 & 9.45 \\
\hline
\end{tabular}

3 


\section{Table 2 (on next page)}

Differentially expressed cuticle genes.

Differentially expressed cuticle genes in T. castaneum larvae injected with CPG dsRNA. Shaded row indicates target gene. 
1 Table 2. Differentially expressed cuticle genes in T. castaneum larvae injected with LOC103313766 cuticle protein dsRNA. Shaded 2 row indicates target gene.

\begin{tabular}{|c|c|c|c|c|c|c|c|}
\hline Gene & LG & $\begin{array}{l}\text { Coordinate } \\
\text { Start }\end{array}$ & $\begin{array}{l}\text { Coordinate } \\
\text { Stop }\end{array}$ & Protein & Annotation & $\begin{array}{l}\text { Fold } \\
\text { Change }\end{array}$ & Expression \\
\hline LOC103313766 & 3 & 4099231 & 4115167 & XP_008196095 & Cuticle protein & 1,491 & $\downarrow$ \\
\hline LOC658110 & 3 & 4097672 & 4112513 & XP_015833062 & Cuticle protein 16.5 & 2.51 & $\downarrow$ \\
\hline LOC658489 & 3 & 4077823 & 4109423 & XP_976285 & Cuticle protein 19.8 & 1.44 & $\downarrow$ \\
\hline LOC 103313752 & 3 & 4114536 & 4126952 & XP_008196069 & Cuticle protein 70 & 10.8 & 个 \\
\hline LOC655183 & 3 & 2446367 & 2620651 & XP_008190752 & Cuticle protein & 4.01 & $\uparrow$ \\
\hline LOC100141875 & 5 & 3608978 & 3616499 & XP_001809825 & Cuticle protein & 16.9 & $\uparrow$ \\
\hline LOC656347 & 5 & 3612511 & 3613210 & XP_008193006 & Cuticle protein 7 & 4.89 & $\uparrow$ \\
\hline LOC107399215 & 2 & 6796409 & 6962942 & XP_015840495 & Larval cuticle protein A2B-like & 25.1 & $\downarrow$ \\
\hline
\end{tabular}

5 


\section{Figure 1}

Venn diagram comparing genes expressed in $T$. castaneum life stages: adult, egg, larvae, and pupae with high expression values ( $>8 \log _{2}$ RPKM).

Shades of grey indicate the relative number of genes in each comparison. Dark grey indicates a higher number of genes and lighter shades represent comparisons with fewer genes.

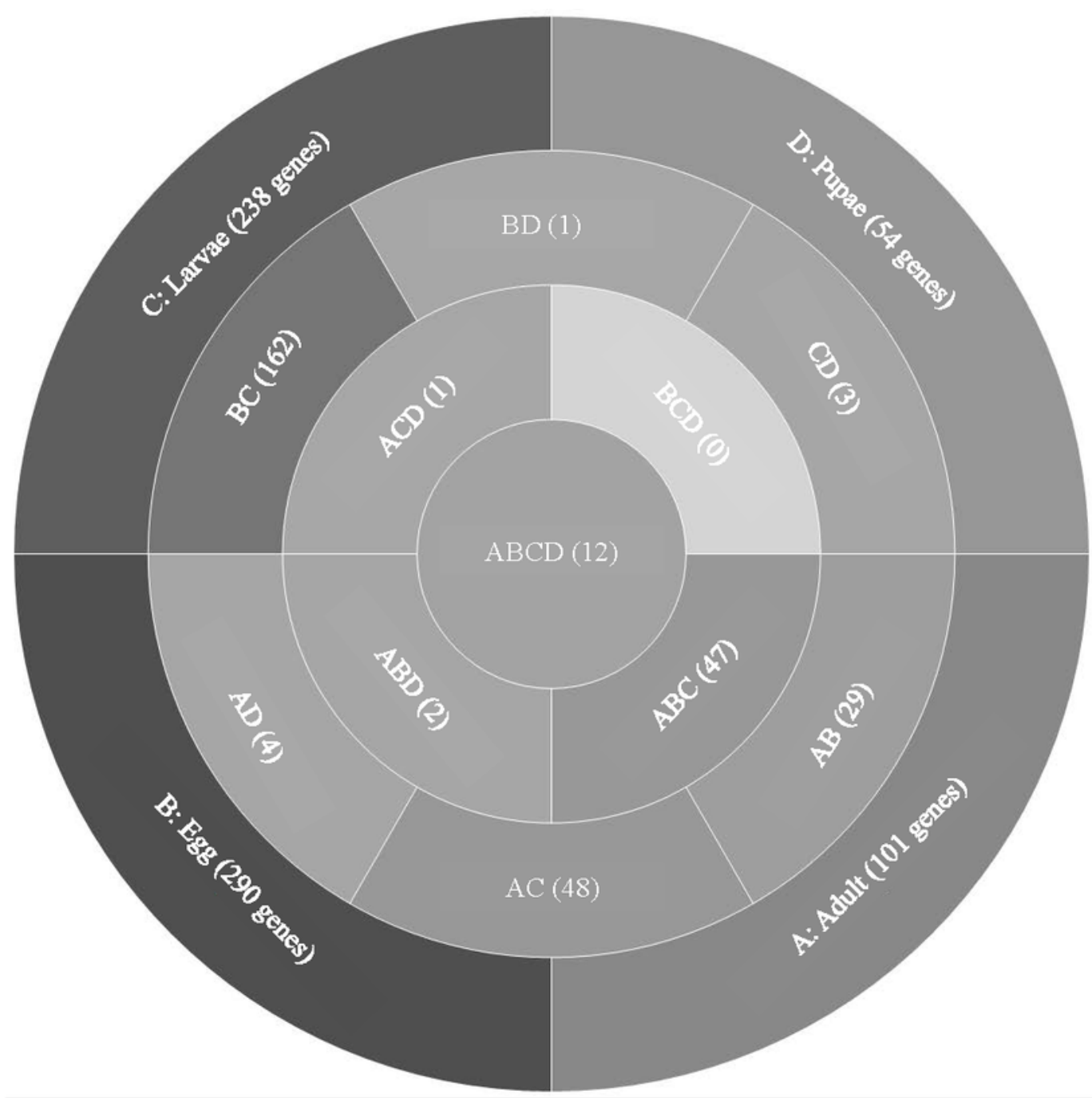




\section{Figure 2}

Top GO terms for genes with high expression in each life stage.

Each pie chart identifies the top GO terms for each life stage; (A) Egg, (B) Larvae, (C) Pupae, (D) Adult.

A. Egg
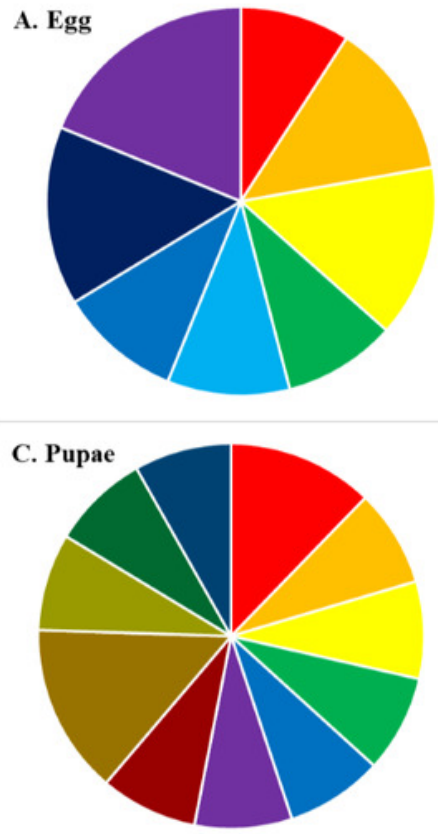

- transferase activity

' metal ion binding

hydrolase activity

" oxidoreductase activity

- protein binding

- ATP binding

- RNA binding

- structural constituent of ribosome

purine ribona cleoside triphosphate binding

structural constitueat of cutick

- metal tou binding

- parime ribonacleotide binding

- transmembrane transporter activily

- parine ribona cleoswe binding

- oxis oreductase acti ity

- peptidase activity

- substrate-specific transporter activity

- tran slation factor activity, RNA binding
B. Larvae

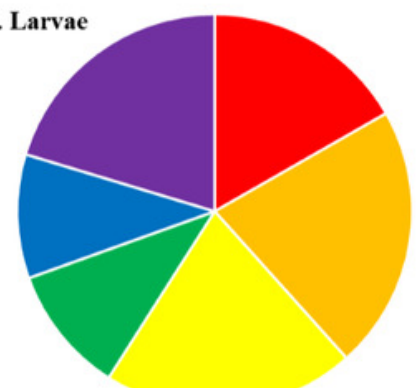

D. Adult

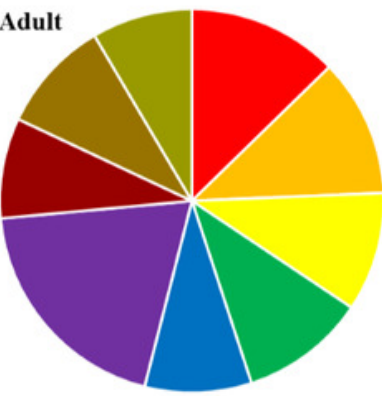

- metal ion binding

hydrolase activity

oxidoreductase activity

hydrogen ion transmembrane

transporter activity

- nucleotide binding

- structural constituent of ribosome

- transfer ase activity

" metal ion binding

carbohydrate derivative binding

" nucleic acid binding

- anion binding

- oxidoreductase activity

- peptidase activity, acting on L-amino

acid peptides
" nudeotide binding

" structural constituent of ribosome 


\section{Figure 3}

Venn diagram comparing genes between all $T$. castaneum life stages: adult, egg, larvae, and pupae with low expression values (2-8 $\log _{2}$ RPKM).

Shades of grey indicate the relative number of genes in each comparison. Dark grey indicates a higher number of genes and lighter shades represent comparisons with fewer genes.

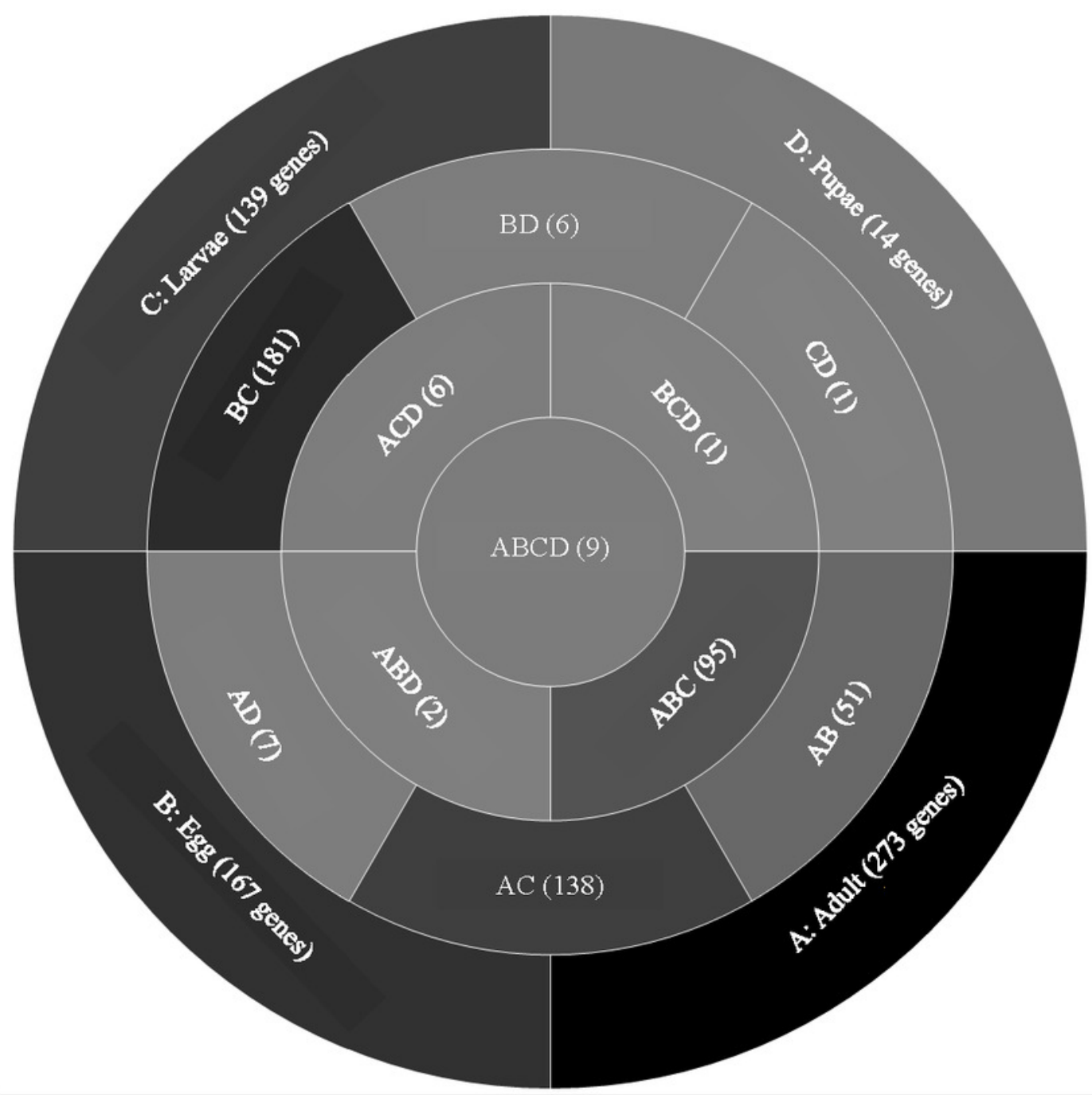




\section{Figure 4}

Top GO terms for genes with low expression in each T. castaneum life stage.

Each pie chart identifies the top GO terms for each life stage; (A) Egg, (B) Larvae, (C) Pupae, (D) Adult.

A. Egg

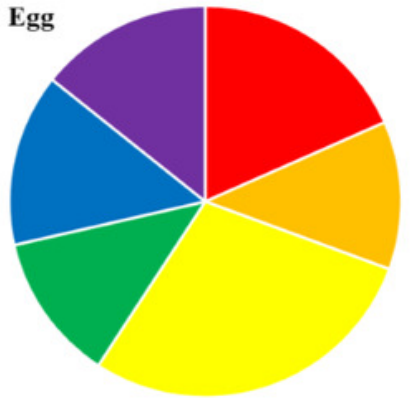

C. Pupae

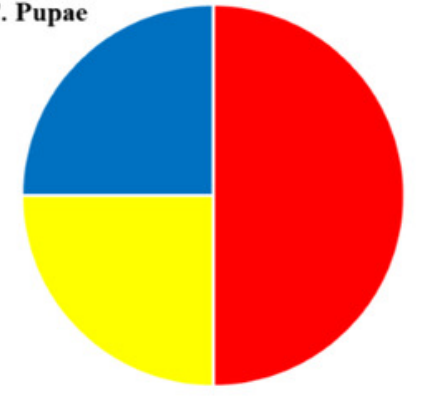

- transcription factor activity, sequence-specific DNA binding - transfer ase activity

sequence-specific DNA binding

- endopeptidase activity

- protein dimerization activity

- zinc ion binding

- structural constituent of cuticle

NADH dehydrogenase (ubiquinone) activity

- serine-type endopeptidase activity
B. Larvae

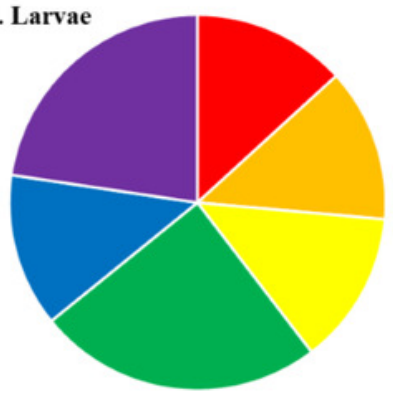

D. Adult

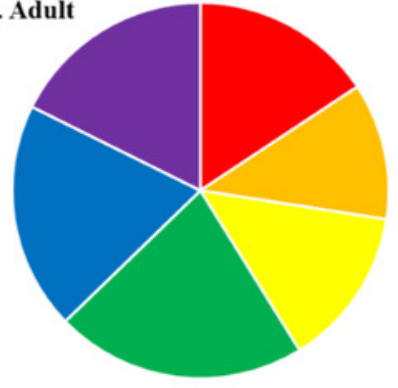

- transition metal ion binding

substrate-specific transmembrane transporter activity

nucleic acid binding

- oxidoreductase activity

- hydrolase activity, acting on ester bonds

- serine-type endopeptidase activity

- transition metal ion binding

nudeoside-triphosphatase activity

transporter activity

- oxidoreductase activity

ATP binding

- serine-type endopeptidase activity 
Figure 5

Effects of injection of CPG dsRNA in T. castaneum larvae.

(A) Average expression of CPG in control, mock, and dsRNA-injected T. castaneum larvae 18 d following RNAi application. (B) Percent survival of $T$. castaneum larvae following RNAi treatment.

A.

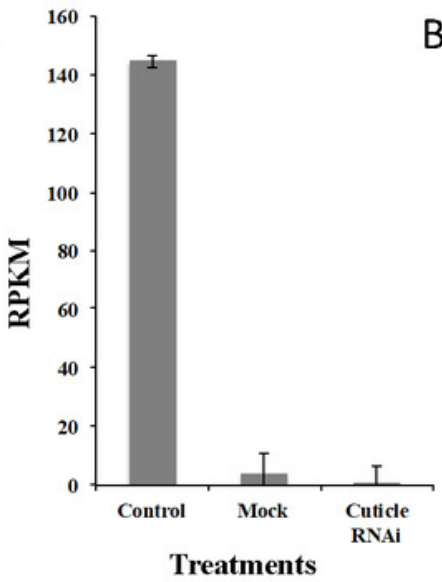

B.

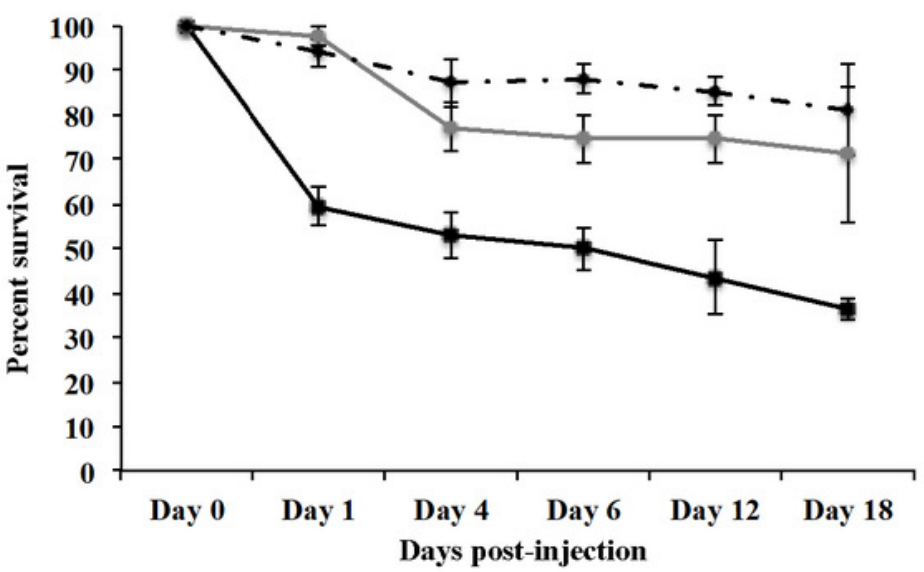


Figure 6

T. castaneum cuticle protein maximum likelihood tree.

CPG (XP_008196095) is highlighted with a yellow box, and proteins from the three cuticle genes with increased expression (XP_008196069, XP_001809825, XP_008190752) are highlighted with a red box. 

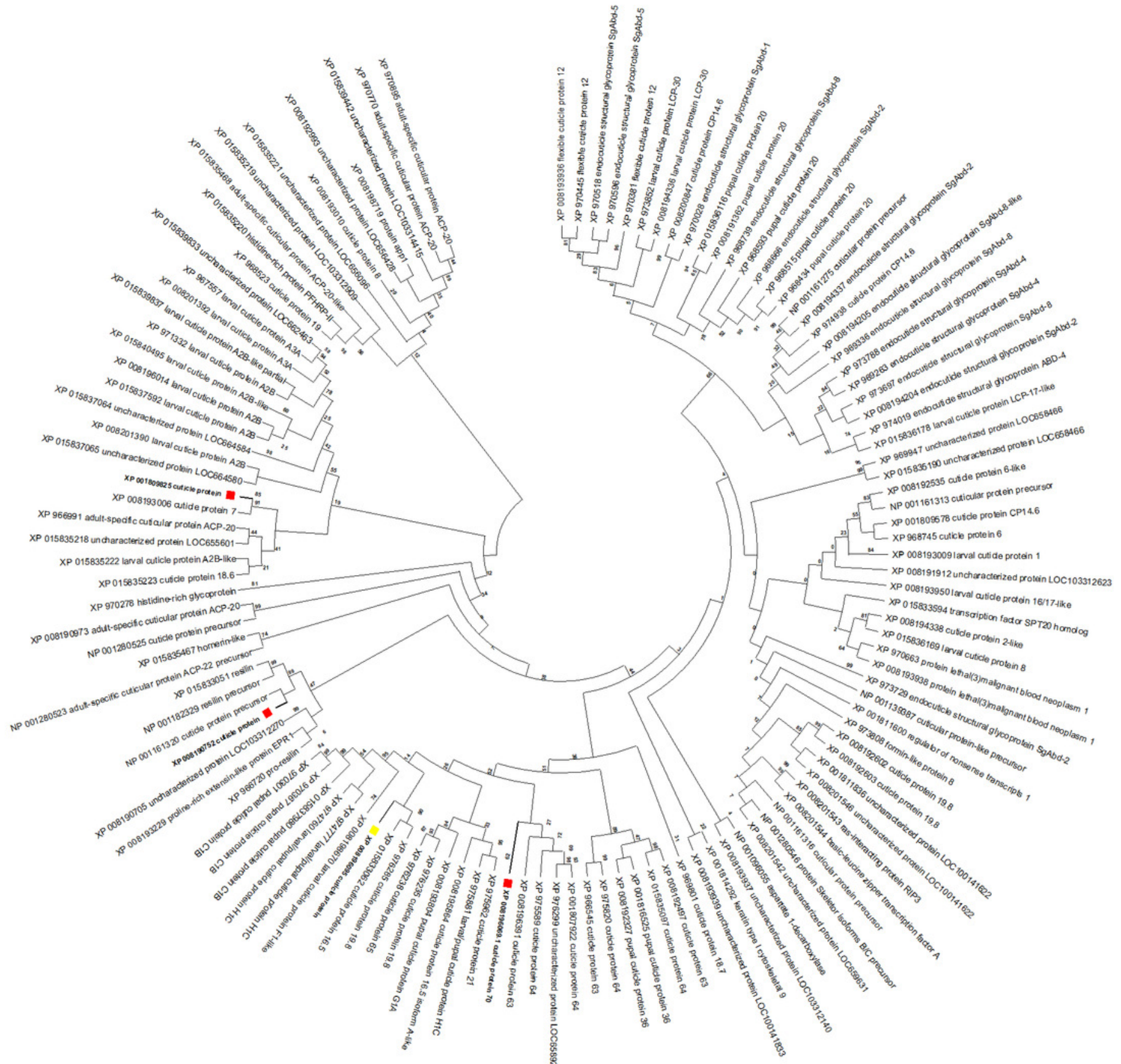